\title{
Comparative study of clinical profile of lean and obese polycystic ovary syndrome women
}

\author{
Akshaya S.*, Ratnaboli Bhattacharya \\ Department of Obstetrics and Gynaecology, Kasturba Hospital, Delhi, India \\ Received: 23 June 2016 \\ Accepted: 28 June 2016 \\ *Correspondence: \\ Dr. Akshaya S, \\ E-mail: akshayadr2010@gmail.com \\ Copyright: () the author(s), publisher and licensee Medip Academy. This is an open-access article distributed under \\ the terms of the Creative Commons Attribution Non-Commercial License, which permits unrestricted non-commercial \\ use, distribution, and reproduction in any medium, provided the original work is properly cited.
}

\section{ABSTRACT}

Background: Polycystic ovary syndrome (PCOS) is the most common endocrine disorder of reproductive-aged women, with an estimated prevalence of $4-8 \%$ and is the most frequent cause of oligoanovulatory infertility. The objective was to compare the clinical profile of PCOS in lean PCOS (body mass index (BMI) $<23 \mathrm{~kg} / \mathrm{m}^{2}$ ) and obese PCOS $\left(\right.$ BMI $\left.>23 \mathrm{~kg} / \mathrm{m}^{2}\right)$ women.

Methods: Fifty healthy women who were euthyroid with age range 15-38 years who presented to gynecology OPD and diagnosed to have PCOS according to ESHRE/ASRM criteria were included in the study. BMI (body mass index) was calculated by the formula weight in $\mathrm{kg}$ / height in meter square. BMI were calculated were divided into 2 groups. Lean PCOS found in patients with $\mathrm{BMI}<23 \mathrm{~kg} / \mathrm{m}^{2}$ and overweight/obese PCOS found in patients with BMI $>23 \mathrm{~kg} / \mathrm{m}^{2}$. History of menstrual irregularity, hirsutism, acne, alopecia, infertility, history of weight gain, history of voice change, family history of PCOS, diabetes were taken. Waist to hip ratio and Ferriman- Gallway score were measured in all patients.

Results: There was no statistically significant difference in the menstrual irregularities, features of hyper androgenism (hirsutism, infertility, acne, alopecia, clitoromegaly) in both lean and obese PCOS. FG score was more in obese PCOS than in lean PCOS. Weight gain, obesity of abdominal type with higher waist to hip ratio $(>0.85)$ were present in all obese as it was the main criteria for group B.

Conclusions: More than half of women with PCOS were obese. The clinical features like menstrual disturbances, infertility, hirsutism, acne and acanthosis nigricans were present in most of PCOS women irrespective of weight. It is noteworthy that even modest weight loss through diet interventions and increased physical activity has favourable effects on reproductive outcome in PCOS.

Keywords: PCOS, Obesity, Infertility, Hyperandrogenism, Menstrual irregularities

\section{INTRODUCTION}

Polycystic ovary syndrome (PCOS) is the most common endocrine disorder of reproductive-aged women, with an estimated prevalence of $4-8 \%$ and is the most frequent cause of oligoanovulatory infertility. ${ }^{1,2}$ The hyperandrogenism clinically manifests as hirsutism, acne and male-pattern baldness, while ovulatory dysfunction may presents as menstrual disturbances, chronic anovulation and infertility. ${ }^{3}$ PCOS is also associated with increased risk of miscarriage. Besides this there is increased risk of glucose intolerance, gestational diabetes, pregnancy induced hypertension and multiple pregnancies.

Obesity, in particular, central obesity plays an important role in development of PCOS. Approximately 50\% of PCOS women are overweight or obese and most of them have the abdominal phenotype. Obesity may play a pathogenetic role in the development of the syndrome in susceptible individuals. In fact, insulin possesses true gonadotrophic function and increased insulin availability 
at the level of ovarian tissue may favour excess androgen synthesis. Obesity per se is a disease entity, and its association with insulin resistance (IR)/hyper insulinemia and metabolic syndrome is well established. Many aspect of insulin action in obesity resembles those seen in PCOS. $^{4}$

Increased adiposity is associated with several abnormalities of sex steroid metabolism and results in increased androgen production and suppression of SHBG. ${ }^{5}$ Furthermore, obese patients with PCOS have more severe cardiometabolic risk factors, compared with their lean counterparts. ${ }^{6}$

We sought to thereby gain insight into possible pathogenetic differences between non obese and obese subgroups of women with PCOS and its resultant clinical heterogeneity. The objective was to study and compare the clinical profile of PCOS in lean PCOS (BMI $<23 \mathrm{~kg} / \mathrm{m}^{2}$ ) and obese PCOS women (BMI $>23 \mathrm{~kg} / \mathrm{m}^{2}$ ).

\section{METHODS}

This observational cross sectional study was conducted in the department of gynaecology at Kasturba hospital, Delhi, India. Total fifty patients who attended gynecology OPD and met the inclusion criteria were enrolled in the study. BMI were calculated were divided into 2 groups.

Group A (lean PCOS): Patients with BMI $<23 \mathrm{~kg} / \mathrm{m}^{2}$.

Group B (overweight/obese PCOS): Patients with BMI $>23 \mathrm{~kg} / \mathrm{m}^{2}$

Women of age range 15-38 years diagnosed to have PCOS according to ESHRE/ASRM criteria were included in the study after obtaining a written informed consent.

\section{Exclusion criteria}

Other causes of hyperandrogenism, patient who have received any medication known to affect carbohydrate metabolism for at least 3 months before the study, women using oral contraceptive pills, pregnancy and lactation and hypothyroidism.
Patients were examined and investigated after a detailed history. History of menstrual irregularity, hirsutism, acne, alopecia, infertility, history of weight gain, history of voice change, family history of PCOS, diabetes were taken. Waist to hip ratio and Ferriman-Gallway score were measured in all patients.

\section{Statistical evaluation}

Statistical evaluation was done by using SPSS (statistical package of the social sciences). Qualitative variables were expressed as numbers and percentages while quantitative variables were expressed as means. P-value less than 0.05 was considered as significant.

\section{RESULTS}

Out of 50 women were diagnosed to have PCOS according to ESHRE/ASRM criteria, there were more obese or overweight women $(56 \%)$ with $\mathrm{BMI}>23$ and mean BMI of $29.52 \pm 2.43$. There were $44 \%$ who were lean with $\mathrm{BMI}<23$ and mean BMI of lean 21.87 \pm 1.19 .

The difference in the age in both groups was statistically significant $\mathrm{p}$-value $=0.029$ (statistically significant) by fisher exact test. Hence obese PCOS usually presents in older age group. Obese PCOS belonged to higher socioeconomic status than the lean. $46 \%$ of women were nulliparous.

The women with PCOS came with presenting complaints as, menstrual irregularity being commonest in 47 (94\%) women followed by hirsutism in $42(84 \%)$ women. Infertility was present in $21(42 \%)$. Obesity and weight gain was present in $28(56 \%)$ and $15(30 \%)$ respectively. $9(18 \%)$ women presented with acne. There was no statistically significant difference in the menstrual irregularities, features of hyperandrogenism (hirsutism, infertility, acne, alopecia, clitoromegaly) in both lean and obese PCOS. Since obesity is a criterion to diagnose group B women none of the group A (lean PCOS) presented with history of weight gain. The most common presentations were menstrual irregularities in the amenorrhea, oligomenorrhoea, polymenorrhoea, and intermenstrual bleeding irrespective of their weight (Table 1).

Table 1: Distribution of women with PCOS according to presenting complaints.

\begin{tabular}{|c|c|c|c|c|c|c|c|}
\hline \multirow{2}{*}{ Presenting complaints } & \multicolumn{2}{|l|}{ Total } & \multicolumn{2}{|c|}{ Group A (lean PCOS) } & \multicolumn{2}{|c|}{ Group B (obese PCOS) } & \multirow[t]{2}{*}{ P-valut } \\
\hline & Number & $\%$ & Number & $\%$ & Number & $\%$ & \\
\hline Menstrual irregularity & 47 & $94 \%$ & 20 & $90.9 \%$ & 27 & $96.4 \%$ & 0.576 \\
\hline Hirsutism & 42 & $84 \%$ & 18 & $81.8 \%$ & 24 & $85.7 \%$ & 0.718 \\
\hline Infertility & 21 & $42 \%$ & 10 & $45.4 \%$ & 11 & $39.2 \%$ & 0.661 \\
\hline Acne/oily skin & 9 & $18 \%$ & 2 & $9.1 \%$ & 7 & $25 \%$ & 0.266 \\
\hline Weight gain & 15 & $30 \%$ & 0 & $0 \%$ & 15 & $53.6 \%$ & $<0.001$ \\
\hline Obesity & 28 & $56 \%$ & 0 & $0 \%$ & 28 & $100 \%$ & $<0.001$ \\
\hline
\end{tabular}


There was a more significant association of family history of PCOS and diabetes in obese than in lean. The mean weight in lean PCOS was $47 \mathrm{~kg}$ whereas the mean weight in obese PCOS was $66.78 \mathrm{~kg}$. The difference of weight was statistically significant between the two groups (Table 2).

The mean waist to hip ratio of all the PCOS women was $0.90 \pm 0.12$. The mean waist to hip ratio in lean PCOS was $0.82 \pm 0.02$ whereas the mean waist to hip ratio of obese PCOS was $0.97 \pm 0.12$ (Table3).
Table 2: Family h/o of PCOS and diabetes.

\begin{tabular}{|lccccc|}
\hline History & Number & $\%$ & Number & $\%$ & $\begin{array}{l}\text { p } \\
\text { value }\end{array}$ \\
\hline $\begin{array}{l}\text { Family } \\
\text { h/o of }\end{array}$ & 1 & 4.5 & 11 & 39.3 & $<0.001$ \\
\begin{tabular}{l} 
PCOS \\
\hline $\begin{array}{l}\text { Family } \\
\text { h/o of } \\
\text { diabetes }\end{array}$
\end{tabular} & 1 & 4.5 & 10 & 37.7 & 0.04 \\
\hline
\end{tabular}

Table 3: Waist to hip ratio in women with PCOS.

\begin{tabular}{|lllllll|}
\hline Waist to hip ratio & Total & & \multicolumn{2}{c|}{ Group A (lean PCOS) } & \multicolumn{2}{c|}{ Group B (obese PCOS) } \\
\hline & Number & $\%$ & Number & \% & Number & \% \\
\hline$<0.85$ & 21 & $42 \%$ & 21 & $95.5 \%$ & 0 & $0 \%$ \\
\hline$>0.85$ & 29 & $58 \%$ & 1 & $4.5 \%$ & 28 & $100 \%$ \\
\hline Mean WHR & $0.90 \pm 0.12$ & & $0.82 \pm 0.02$ & & $0.97 \pm 0.12$ & \\
\hline
\end{tabular}

Table 4: Features of hyperandrogenism.

\begin{tabular}{|c|c|c|c|c|c|c|c|}
\hline \multirow{2}{*}{ Hyper androgenism } & \multicolumn{2}{|l|}{ Total } & \multicolumn{2}{|c|}{ Group A (lean PCOS) } & \multicolumn{2}{|c|}{ Group B (obese PCOS) } & \multirow[t]{2}{*}{ P-value } \\
\hline & Number & $\%$ & Number & $\%$ & Number & $\%$ & \\
\hline Hirsutism & 42 & $84 \%$ & 18 & $81.8 \%$ & 24 & $85.7 \%$ & 0.718 \\
\hline Acne & 9 & $18 \%$ & 2 & $9.1 \%$ & 7 & $25 \%$ & 0.266 \\
\hline Alopecia & 2 & $4 \%$ & 0 & $0 \%$ & 2 & $7.1 \%$ & 0.497 \\
\hline Clitoromegaly & 1 & $2 \%$ & 0 & 0 & 1 & $3.6 \%$ & 1.00 \\
\hline Change in voice & 0 & $0 \%$ & 0 & $0 \%$ & 0 & $0 \%$ & 1 \\
\hline Acanthosis & 6 & $12 \%$ & 2 & $9.1 \%$ & 4 & $14.3 \%$ & 0.683 \\
\hline
\end{tabular}

Table 5: Ferriman Gallway score in hirsutism.

\begin{tabular}{|lllllll|}
\hline \multirow{2}{*}{ Hirsutism score } & Total & & Group A (lean PCOS) & \multicolumn{2}{l|}{ Group B (obese PCOS) } & P-value \\
\cline { 2 - 7 } & No & $\mathbf{\%}$ & No & \% & No & \% \\
\hline Absent $(<8)$ & 8 & 16.0 & 4 & 18.2 & 4 & 14.3 \\
\hline Mild $(9-16)$ & 24 & 48.0 & 18 & 81.8 & 6 & 21.4 \\
\hline Moderate $(17-25)$ & 15 & 30.0 & 0 & 0.0 & 15 & 53.6 \\
\hline Severe $(>25)$ & 3 & 6.0 & 0 & 0.0 & 3 & 10.7 \\
\hline Mean score & $13.08 \pm 6.48$ & $8.59 \pm 1.94$ & & $16.61 \pm 6.64$ & \\
\hline
\end{tabular}

There was no difference in features of hyperandrogenism in both groups as evident by hirsutism, acne, alopecia, clitoromegaly, acanthosis, change in voice (Table 4). Although the incidence of hirsutism was similar in obese and non-obese but its severity as judged by Ferriman Gallway score was more in obese women.

\section{DISCUSSION}

The complexity and diversity of both underlying pathology as well as clinical manifestations renders difficulty in defining better predictors of PCOS. Most of the women $(74 \%)$ with PCOS belonged to age group 20-30 years, out of these $17(77.3 \%)$ were lean and $20(71.4 \%)$ were obese PCOS. Obese PCOS presented in older age group. PCOS has its origin during adolescence and its metabolic abnormalities worsen as the age increases with resultant obesity. Menstrual disturbance was the most common presenting complaint (94\%) in the women with PCOS. The presentation of menstrual irregularities was comparable in obese $(96.4 \%)$ and lean group (90.9\%) in this study comparable to study done by Saxena $\mathrm{P}$ et al. $^{7}$ Hirsutism was the next most common presenting symptom present in $84 \%$ of PCOS. The incidence of hirsutism didn't differ much between the lean $(81.8 \%)$ and obese PCOS $(85.7 \%)$. Goldzeiher et al reported the incidence of hirsutism in PCOS women as $69 \%$ which is comparable with present study. ${ }^{8}$ In the study by Franks et al found that $42 \%$ of PCOS women came with infertility which is comparable with result of present study. ${ }^{9}$ Acne was a presenting complaint in $18 \%$ of subjects and did not differ in obese and lean group. In the present study, since obesity is a criterion to diagnose group B women none of the group A (lean PCOS) 
presented with history of weight gain. Obesity was the $3^{\text {rd }}$ most common presenting complaint. $56 \%$ of women had obesity in this study. All 28 PCOS women were obese in group $\mathrm{B}$ as it was a selection criterion for them. Obese PCOS were more common than lean PCOS in this study as well as study by Saxena $\mathrm{P}$ et al. ${ }^{7}$ The presence of family history of PCOS and obesity was seen to be more in obese group than in lean group. In the present study, $29(58 \%)$ PCOS women had WHR >0.85 (central obesity) which included all $28(100 \%)$ obese women and $1(4.5 \%)$ lean PCOS women. Holte J, reported the mean waist to hip ratio in lean PCOS as 0.79 and mean waist to hip ratio of obese PCOS as $0.90 .{ }^{10}$ It was noted that central obesity is more common in obese PCOS than the lean PCOS woman which is comparable to present study. Even though the hirsutism was comparable in both groups, however the degree of hirsutism was exaggerated in obese. $4 \%$ of PCOS came with alopecia and all of them were obese. The presence of alopecia was comparable in both groups. $6 \%$ of PCOS women had alopecia in the study by Balen et al which is comparable with our result. Only one obese PCOS (3.6\%) presented with clitoromegaly. ${ }^{11}$ The presence of clitoromegaly was comparable in both the groups. In the study by Saxena $\mathrm{P}$ et al in found that $2.3 \%$ of lean and $3.4 \%$ of obese PCOS had clitoromegaly which is comparable to this study. ${ }^{7}$ Acanthosis nigricans was present in $12 \%$ women. This condition is a sign of insulin resistance. Insulin spillover into the skin results in hyperplasia, an abnormal increase in skin growth. It was comparable in both groups.

\section{CONCLUSION}

PCOS, an ill-defined symptom complex need its due attention. More than half of the women were obese. The clinical features like menstrual disturbances, infertility, hirsutism, acne, and acanthosis nigricans were present in most of PCOS women irrespective of weight. Even though the hirsutism was comparable in both groups, however the degree of hirsutism was exaggerated in obese as noticed by higher FG score. Women presenting with oligomenorrhoea should be further investigated for PCOS and treated accordingly. Central obesity was more common in obese PCOS than the lean PCOS. Therapeutic timely intervention can halt this on-going process.

Funding: No funding sources Conflict of interest: None declared

Ethical approval: The study was approved by the Institutional Ethics Committee

\section{REFERENCES}

1. Knochenhauer ES, Key TJ, Kahsar MM, Waggoner W, Boots LR, Azziz R. Prevalence of polycystic ovary syndrome in black and white women of southeastern united states: prospective study. J Clin Endocrinol Metab. 1998;83:3078-82.

2. Hull MG. Epidemiology of infertility and polycystic ovarian disease: endocrinological and demographic studies. Gynecol endocrinol. 1987;1:235-45.

3. Simpsons JL, elucidating the genetics of PCOS. Dunaif A, Givens JR, Haseltine FP, Merriam GR. Current issues in endocrinology and metabolism: polycystic ovary syndrome. Boston: Blackwell scientific; 1992:59-69.

4. Kopelman PG. Hormone and obesity. Clin Endocrino Metab. 1994;8:549-75.

5. Pasquali R. Obesity and androgens: facts and perspectives. Fertil steril. 2006;85:1319-40.

6. Cattrall FR, Healy DL. Long term metabolic, cardiovascular and neoplastic risks with polycystic ovary syndrome. Best Pract Res Clin Obstet Gynaecol. 2004;18:803-12.

7. Saxena P, Prakash A, Nigam A, Mishra A. PCOS: is obesity a sine qua non? a clinical, hormonal, and metabolic assessment in relation to BMI. Indian $\mathbf{J}$ Endocrinol Metab. 2012;16(6):996-9.

8. Goldzieher JW, Axelrod LR. Clinical and biochemical features of polycystic ovarian disease. Fertil Steril. 1963;14:631-53.

9. Adams J, Polson DW, Franks S. Prevalence of polycystic ovaries in women with anovulation and idiopathic hirsutism. British Med J. 1986;293:355-9.

10. Holte, Bergh T, Bernet C, Lithell C. Serum lipoprotein lipid profile in women with the polycystic ovary syndrome: relation to anthropometric, endocrine and metabolic variables. Clinic Endocrino. 1994;41:463-71.

11. Balen A, Conway G, Klatsas G. Polycystic ovary syndrome: the spectrum of the disorder in 1741 patients. Human Repro. 1995;10:2107-11.

Cite this article as: Akshaya S, Bhattacharya R. Comparative study of clinical profile of lean and obese polycystic ovary syndrome women. Int J Reprod Contracept Obstet Gynecol 2016;5:2530-3. 